\title{
UV-Visible Transmittance of Silicone-Hydrogel Contact Lenses measured with a fiber optic spectrometer
}

\author{
R. Fuentes*, E. Fernández, I. Pascual and C. García \\ Dpto. Óptica, Farmacología y Anatomía, Universidad de Alicante, Apartado 99, E-03080 \\ Alicante, Spain \\ Instituto Universitario de Física Aplicada a las Ciencias y las Tecnologías, Universidad de Alicante, \\ Apartado 99, E-03080, Alicante, Spain \\ *fuentes@ua.es
}

\begin{abstract}
Protein deposition is one of the most frequent contaminants occurring on hydrophilic contact lenses and may modify the lens optical properties. The silicone-hydrogel contact lenses usually adsorb a lower amount of proteins than the conventional hydrogel ones. However it is important to study the influence of protein deposits on some silicone-hydrogel contact lenses properties, such as UV-Visible transmittance. In this study it is measured de UV-Visible properties of new and worn silicone-hydrogel contact lenses and the transmittance changes that can occur with wear are quantified.

An optical fiber spectrometer has been used for measuring the transmittance of contact lenses. This spectrometer has the advantage that the lens can be positioned horizontally and the light beam passes through it perpendicularly. For the measurements, it was necessary to make a special cuvette to put inside the lens and keep it submerged in sterile saline solution.

The contact lenses used were Biofinity ${ }^{\circledR}$. They are manufactured by CooperVision with a material whose technical name is Comfilcon A. This material has not yet been analyzed for this purpose. Moreover, in this type of studies are commonly taken lenses of power $-3.00 \mathrm{D}$, however we have not limited to a single power value but we have extended the range to lower and higher values regarding to the standard power in order to see how this parameter affects.
\end{abstract}

Keywords: Silicone-hydrogel contact lenses, optical fiber spectrometer, transmittance.

\section{INTRODUCTION}

The electromagnetic spectrum is a wide array of diverse waves, including short waves, infrared rays, X-rays, and gamma rays. A small part of it is visible spectrum; it is the only portion of the total spectrum we can see. It occupies wavelengths of approximately 400 to $700 \mathrm{~nm}$. To one side of the visible spectrum is the ultraviolet radiation (UVR) and to the other side are the infrared rays. According to the International Commission on Non-Ionizing Radiation Protecction ${ }^{1}$, UVR occupies that portion of the electromagnetic spectrum from al least 100 to $400 \mathrm{~nm}$ and it is subdivided into three bands. The band 100 to $280 \mathrm{~nm}$ is designated as UVC, $280-315 \mathrm{~nm}$ as UVB and 315 to $400 \mathrm{~nm}$ as UVA. This data are provided by Commission on Illumination (CIE).

Wavelengths below $180 \mathrm{~nm}$ (vacuum UV) are of little practical biologic significance since they are readily absorbed in air ${ }^{1}$. UVA is the UV radiation nearest to the visible spectrum and is absorbed mostly by the crystalline lens. Thus, the retina is generally protected from UVA radiation. UVB radiation, on the other hand, is absorbed primarily by the cornea and conjunctive, with up to $10 \%$ penetration to the anterior lens surface.

The spectral transmittance properties of contact lenses are particularly important for visual performance level. It is therefore necessary to study these properties and comparing their values when the lenses are new and after they have been worn during the time established by the manufacturer. The optical quality of the lenses will be depend on the absorption characteristics. Previous studies have evaluated the spectral transmittance for a variety of tinted and UVblocking soft lenses ${ }^{2-6}$.

8th Iberoamerican Optics Meeting and 11th Latin American Meeting on Optics, Lasers, and Applications, edited by Manuel Filipe P. C. Martins Costa, Proc. of SPIE Vol. 8785, 8785AZ

(C) 2013 SPIE $\cdot$ CCC code: $0277-786 X / 13 / \$ 18 \cdot$ doi: $10.1117 / 12.2025710$ 
Protein deposition is one of the most frequent contaminants occurring on hydrophilic contact lenses, resulting from the interaction between the tear film and the polymer of a contact lens ${ }^{7-9}$ and may modify the lens optical properties ${ }^{7}$. Protein films form a distinct group of surface deposits, characterized by a thin and semi opaque superficial layered appearance. Although silicone-hydrogel lenses usually adsorb a lower amount of proteins than the conventional hydrogel ones ${ }^{9}$, it is important to study the influence of protein deposits on some silicone-hydrogel contact lenses properties, such as UVVisible transmittance.

Lira et al. ${ }^{10}$ evaluate the influence of wear of silicone-hydrogel contact lenses on lens ultraviolet and visible spectrum transmittance by studying several contact lenses before an after wear. They use a Simadzu UV3101-PC UV-VIS-NIR spectrophotometer. It is a bench-up spectrometer of double beam. They use lenses of different companies as Johnson \& Jonson Vision Care, Inc, Ciba Vision and Bausch \& Lomb. Their study indicate that transmittance is modified after contact lenses wear, probably due to de formation of biofilms on the contact lens surface, being more noticeable in the UVR region of the spectrum. The lenses that do not have UV absorbers incorporated into the polymer transmitted most of the UV radiation (UVR) before and after wear. Silicone-hydrogel and conventional hydrogel contact lens materials that provide UVR protection (UV-blocker) maintain this property even after being worn. It was demonstrated that the transmittance characteristics were strongly dependent on the lens material.

The standard methods for determining the transmission or absorption spectrum of contact lenses generally involve the use of a bench-top spectrometer with the test lens mounted in an adapted cuvette placed in the fixed light path of the system. Spectrometer beam size is determined by slit width and height and the angular divergence of the coupling optics extended over the relatively large path length. The spectrometer's light beam therefore may be incident on relatively broad area of the test lens, so a large sample lens area transmission is measured, although it may vary across its diameter because of the thickness differences that provide power. For example a $-3.00 \mathrm{D}$ lens with a central thickness of $0.08 \mathrm{~mm}$ will increase to approximately twice that value at the periphery and the converse will be true for a $+3.00 \mathrm{D}$ lens.

However when it is used a fiber optic spectrometer it is achieved illuminate a smaller area, whereby the value obtained by measuring the transmittance is more accurate. Walsh ${ }^{11}$ used a fiber optic spectrophotometer to measure lens transmission curves at test points across lens surfaces to determine optical properties based on the Beer-Lambert law. To quantify the spatially specific transmission spectra across a number of the test lenses, an Ocean Optics S2000 miniature fiber optic spectrophotometer with a spectral range of 200 to $800 \mathrm{~nm}$ and spectral bandwidth of $3 \mathrm{~nm}$ was used. Factors determining the transmission curves include the hydrogel lens used, its refractive index whether a UV radiation-blocking dopant is incorporated, the water content, and the thickness of the lens. He showed that UV-radiation blocking hydrogel contact lenses provide protection across the ocular surface, which varies according to the lens thickness. Transmission curves across the lenses showed greater UV radiation-blocking capacity at the thicker peripheral region. In addition, the ability to determine the spatially specific absorption coefficient and related UV radiation protection factor was demonstrated. Therefore in this study Walsh ${ }^{11}$ showed that the measurement system it could determine the spatial variation of the transmission of the lens. This method was more accurate than standard methods that provided the benchup spectrometers of double beam. He recognizes the importance of maintaining the contact lenses with a proper level of hydration during the time of performing the test, because the value of the transmittance depends on this factor.

Since it has been demonstrated that the transmittance characteristics are strongly dependent on the lens material, the objective of this work was to evaluate the influence of wear of silicone-hydrogel contact lenses Biofinity (Cooper Vision) on ultraviolet and visible spectrum transmittance by studying several contact lenses before an after wear. These contact lenses are manufactured from a material called Comfilcon A. Since their launch in 2007 no study has been presented for this purpose. The transmittance of this contact lenses has never been reported before and neither its variation with the use. Moreover in this type of studies are commonly taken lenses of power $-3.00 \mathrm{D}$, however we have performed a study for an extensive range of powers, ranging from $-1.50 \mathrm{D}$ to $-7.50 \mathrm{D}$.

The studied range of wavelengths covers from $300 \mathrm{~nm}$ to $700 \mathrm{~nm}$, that is, UVB, UVA and visible spectrum. The UVC transmittance is not considered, since any ocular health concerns. Only small amounts of radiation in the UVC range actually reach the earth's surface.

In order to measure the transmittance we have used a fiber optic spectrometer technique that provides an accurate measure because it illuminates a small area of the lens where the thickness can be considered constant. Further measurements are made with the lens immersed in saline solution inside cuvette and this allows keeping its normal hydration level always. 


\section{MATERIAL AND METHODS}

\subsection{Contact Lenses}

Silicone hydrogel contact lenses are named for the materials used to make these lenses. These contact lenses are the latest kind of soft lenses commercially available Contact lenses have progressed as people develop better technology and materials. Soft contact lenses were made possible in the 1960s and 1970s by the development of polymers that could absorb water: large molecules made of repeated pieces. These were called "hydrophilic gels" or simply "hydrogels". Silicone hydrogels are the latest in a line of developments aimed at increasing the oxygen permeability (increased comfort, longer wear and better eye health), wettability, and clinical performance of contact lenses. Silicone has higher oxygen permeability, allowing more oxygen to pass, than water, so oxygen permeability is no longer tied to how much water is in each lens. This type of lens provides excellent oxygen transmissibility to the cornea on account of silicone's high oxygen permeability when compared to the conventional hydrogel contact lenses ${ }^{12,13}$.

There are many types, even generations, of silicone hydrogels used to manufacture contact lenses today. These come with technical names such as Galyfilcon, Senofilcon, Comfilcon and Enfilcon. These materials are each used to make contact lenses that address different corrective and wearing needs. Each contact lens, whatever the material, is U.S. FDA-approved or cleared for specific wear and replacement conditions.

The primary benefit of silicone hydrogel lenses is that they reduce the tradeoff between oxygen permeability and wettability. This opens up many possibilities for silicone hydrogel contacts, including: extended wear (sometimes for up to six straight nights and days), continuous wear (sometimes for up to thirty days before replacement) and increased comfort and performance.

Contacts lens transmission curves are determined by factors such as the type of hydrogel material used, whether a UV radiation blocking dopant is incorporated, the water content, and the thickness of the lens.

The characteristics of the contact lenses used in this study are shown in Table I. The contact lenses power ranges between $-1.50 \mathrm{D}$ and $-7.50 \mathrm{D}$.

Table 1. Silicone-hydrogel contact lenses properties used in this study

\begin{tabular}{|c|c|}
\hline Manufacturer & CooperVision \\
\hline Trademark & Biofinity $^{\circledR}$ \\
\hline Material (USAN) & Comfilcon A \\
\hline Water content $(\%)$ & $48 \%$ \\
\hline Central thickness (-3.00 D) & $0.08 \mathrm{~mm}$ \\
\hline Diameter & $14 \mathrm{~mm}$ \\
\hline $\mathbf{R b}$ & $8.6 \mathrm{~mm}$ \\
\hline Surface treatment & No \\
\hline Refractive index & $1.336^{\mathrm{a}}$ \\
\hline Visibility tint & Yes \\
\hline UV blocker & No \\
\hline
\end{tabular}

A total of 26 lenses were measured, first when they were new, unworn, and after when they had been worn. The experiments were performed on contact lenses worn by a group of 13 subjects of both sexes whose ages were between 19 and 22 years. The subjects were informed of the proposed procedure and signed a consent form in accordance with the Helsinki Declaration an full ethics approval was obtained. 
The subjects were instructed to wear their lenses on a daily wear schedule for 6-8 hours and all patients used the same multipurpose solution, Hidro Health $\mathrm{SiH}$ (DISOP S.A.) to store the lenses overnight. Its composition is sodium chloride, monosodium phosphate, sodium phosphate, sodium citrate, poloxamer, polyethylene glycol hydroxystearate, edetate disodium $0.02 \%$ and polyhexamethylene biguanide $0.0002 \%$.

Patients were subjected to tests of biomicroscopy and checking their monocular and binocular visual acuity. This examination was performed to verify if the value of visual acuity changed after contact lens wear.

The contact lenses were replaced after 30 day, according to the manufacturer's instruction. During the test period, there were no complications or signs of discomfort. At the end of the wearing period, each lens was aseptically removed from the eye of the volunteer and placed in a sterile vial containing a saline solution $(0.9 \% \mathrm{NaCl})$. The transmittance of the contact lenses was immediately measured in our laboratory in order to avoid degradation of the contact lenses.

\subsection{Instrumentation and experimental procedure}

To obtain the specific transmission spectra of test lenses, a StellarNet-BLK-C-SR miniature fiber optic spectrometer with spectral range of 200 to $1080 \mathrm{~nm}$ and spectral bandwidth of $1 \mathrm{~nm}$, was used ${ }^{14}$. This instrument is exceptionally robust with no moving parts and is packaged in a small metal enclosure for a variety of spectroscopy applications in field, research lab and factory process. The spectrometer utilizes a $40 \mathrm{~mm}$ diameter concave grating with aberration correction to provide superb spectral imaging because a flat field is projected onto the detector array directly without mirrors yielding uniform resolution across the wavelength range with the lowest possible stray light. The instruments optical input is a standard SMA-905 connector, using single strand fiber optic cable with typical silica core diameters of 400 $\mu \mathrm{m}, 600 \mu \mathrm{m}$ and $1000 \mu \mathrm{m}$. In order to precisely control optical resolution, a slit is permanent installed in the fiber optic connector. This allows the instrument to maintain resolution when different sizes of fiber optic cables are connected.

Optical fibers input and output were connected to a transmission fixture and within this accessory was placed the cuvette with contact lens to be tested. The illuminating optical fiber was coupled to the light source, which could be a deuterium lamp or a halogen lamp and output optical fiber was coupled to the spectrometer. A picture of the test setup is shown in Figure 1.

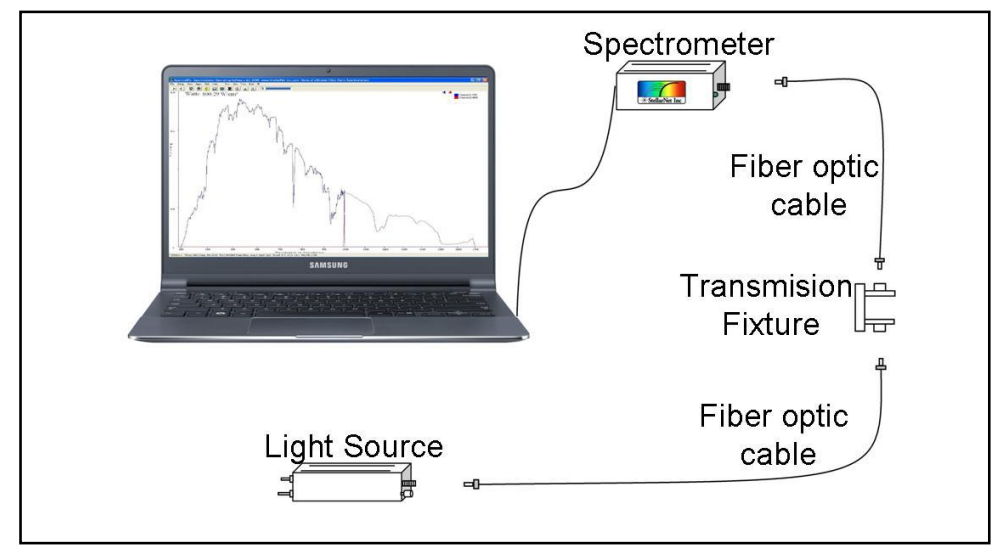

Figure 1: Test setup. Spectrometer and accessories connected.

It was necessary to make a special cuvette for introduce into it the contact lens and it may be immersed in saline solution for the test. The cuvette was made of plastic (polyvinyl chloride, PVC) with a central aperture of $145 \mathrm{~mm}$ since the contact lens was $140 \mathrm{~mm}$ in diameter. The base was a glass sheet with $1 \mathrm{~mm}$ thickness and refractive index of 1.52 . In this way the light beam out of the optical fiber could illuminate a central area of the contact lens with a diameter of $4 \mathrm{~mm}$. The illumination-detection angles are normal to the lens surface. 
Some authors ${ }^{11}$ take the measurement of transmittance without using cuvette to immerse the lens but it has the problem that the experimental measurements can be affected by variations in the test lens transmission related to changes in the hydration levels.

The portable spectrometer is connected to a computer (Fig.1) and using the software associated with the spectrometer it is obtained the spectral transmittance of the contact lenses. First, with the light source off a "Dark Scan" is taken, after with the reference in place (cuvette with saline solution but without contact lens) a "Reference Scan" is taken and finally introducing the sample, that is, placing the contact lens inside the cuvette, a "Sample Scan" is taken.

The computer displays percent transmission at pixel-n using the sample, reference and dark data sets:

$$
T_{n}=\left(\frac{\text { sample }_{n}-\text { dark }_{n}}{r e f_{n}-\text { dark }_{n}}\right) \times 100
$$

The set of values of the percent transmission for each pixel provides the full curve of spectral transmittance of the contact lens.

\section{RESULTS}

For each contact lens the spectrum was recorded three times. Mean transmittance and Median Absolute Deviation (MAD), as a measure of statistical dispersion for each wavelength of every spectral range, were calculated for worn lenses and compared with the value obtained for the same unworn contact lens.

As examples we have chosen four contact lenses with four different powers of the full range we have studied. The transmittance spectra in the range 300 to $800 \mathrm{~nm}$ are displayed in Fig. 2, 3, 4 and 5, allowing the comparison between the mean transmittance spectrum for each worn lens (empty circles) with the one for the unworn lens (filled circles). The Fig. 2 corresponds to -2.5 D; the Fig. 3 to 4.25 D; the Fig. 4 to -5.25 D and the Fig. 5 to $-7 \mathrm{D}$.

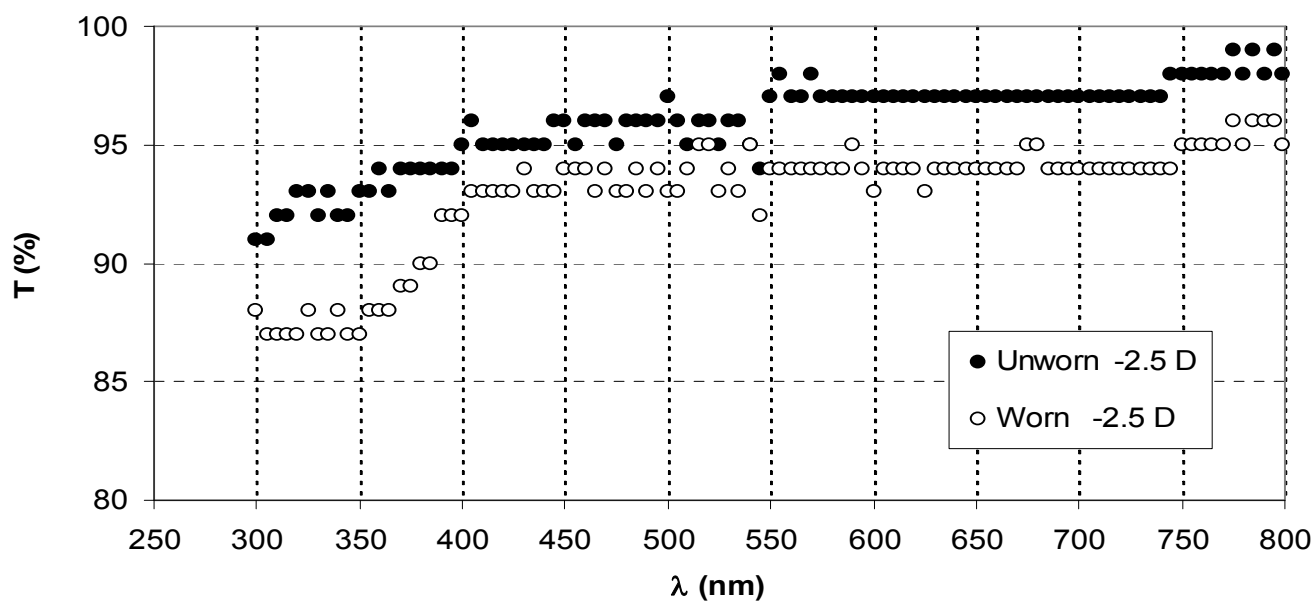

Figure 2. Transmittance spectra for unworn and worn contact lenses of $-2.5 \mathrm{D}$. 


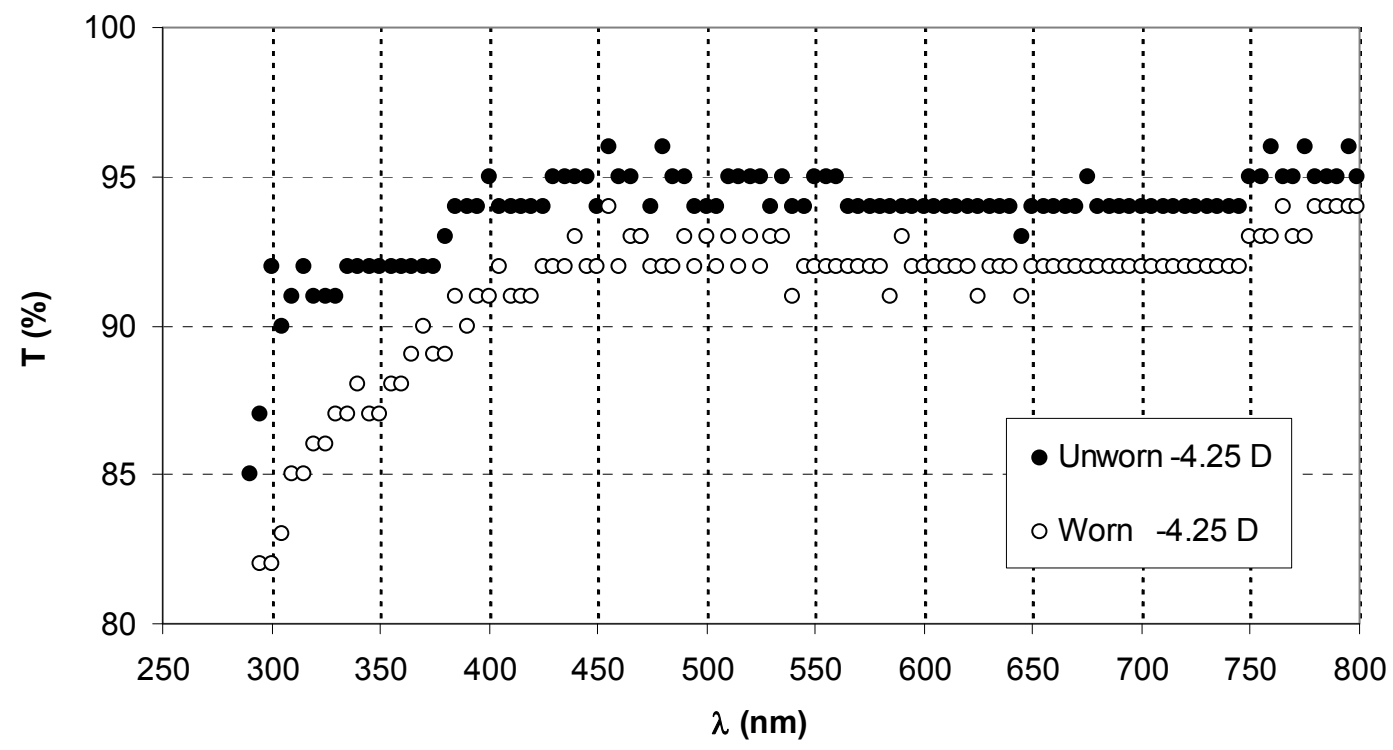

Figure 3. Transmittance spectra for unworn and worn contact lenses of $-4.25 \mathrm{D}$

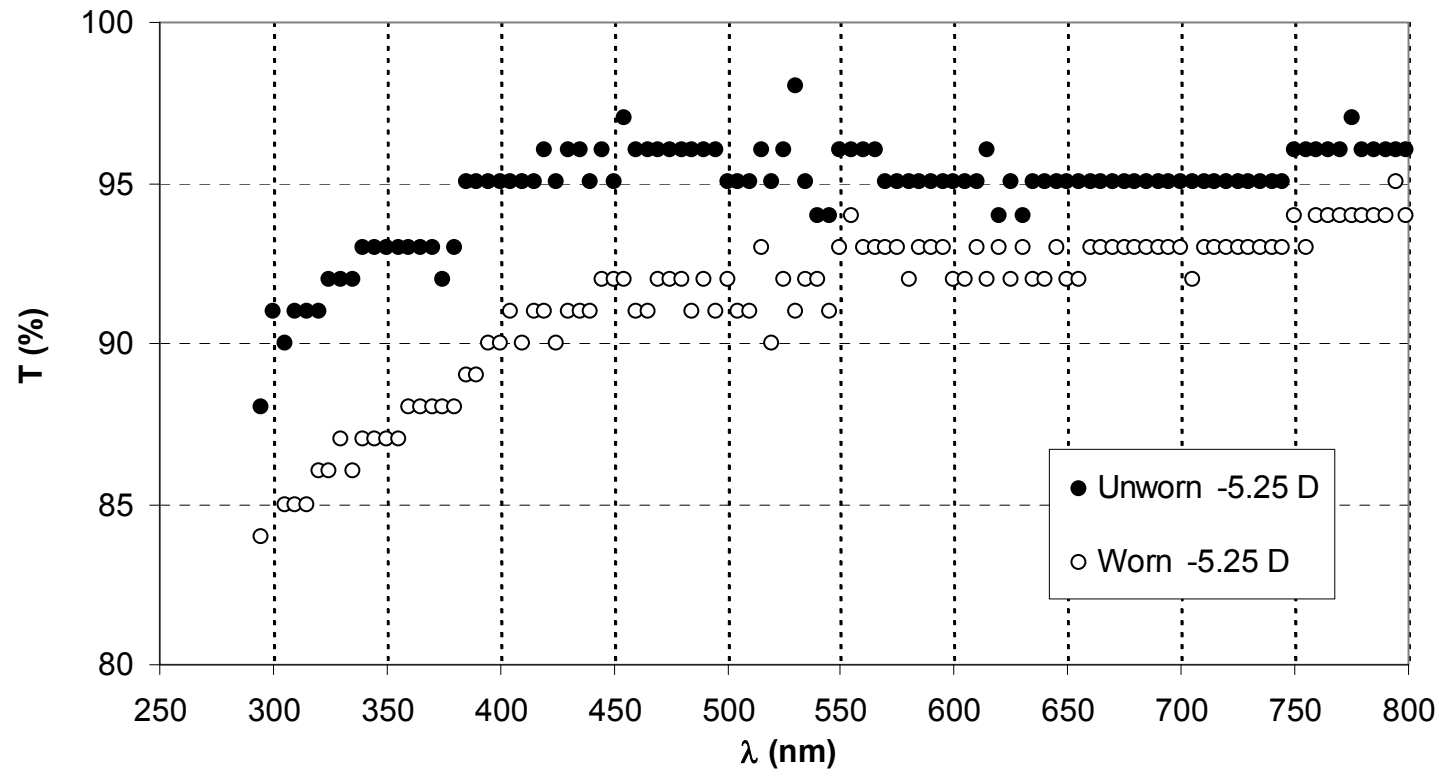

Figure 4. Transmittance spectra for unworn and worn contact lenses of $-5.25 \mathrm{D}$ 


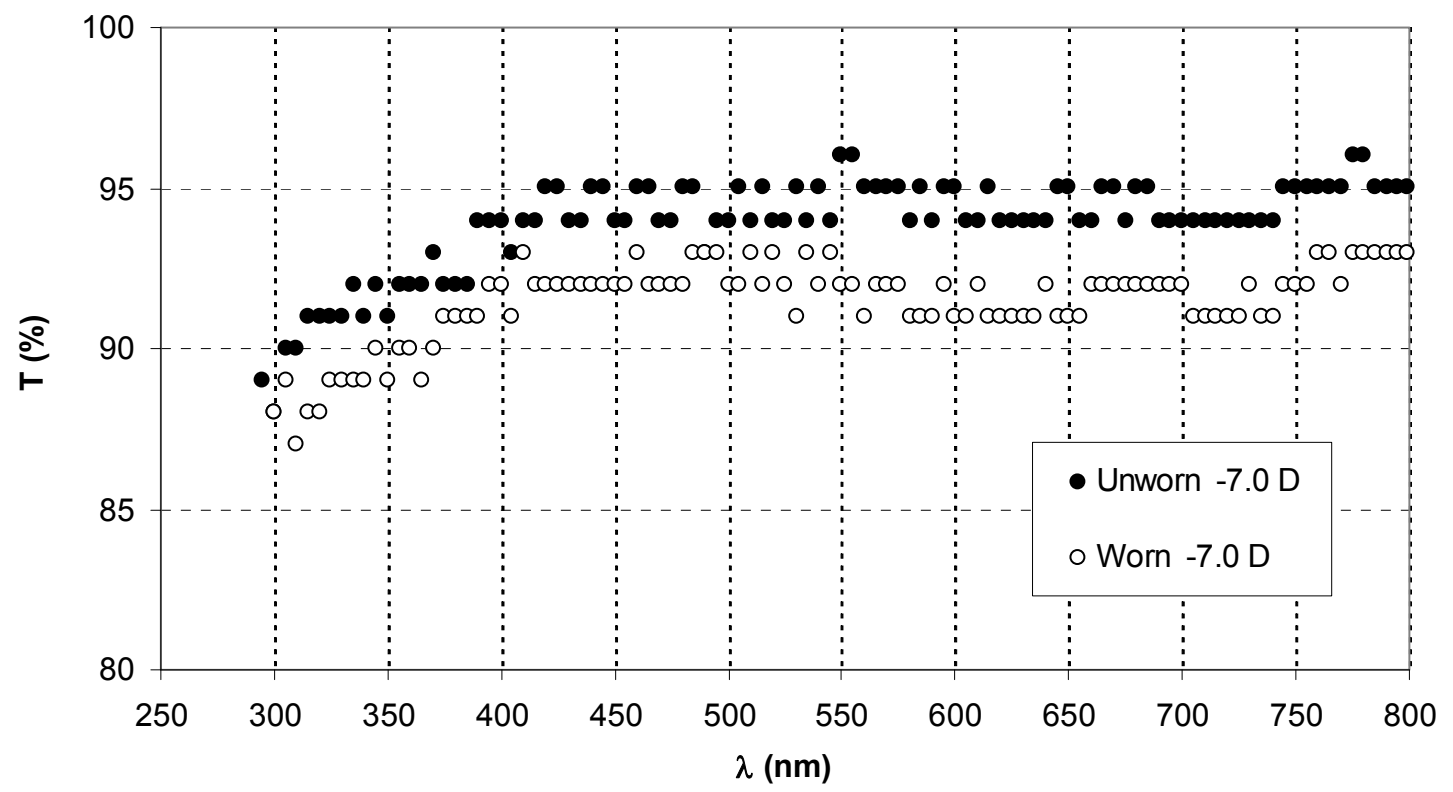

Figure 5. Transmittance spectra for unworn and worn contact lenses of $-7.0 \mathrm{D}$

In general, both in the ultraviolet range and in the visible, slight decrease in transmittance are observed for the worn contact lenses. In Figures 2, 3 and 4 one can see that in the range of UVB waveband, the transmittance is decreased slightly greater than in the visible range. However in Figure 5 this decrease is similar in all spectral ranges.

Table 2 shows the results obtained for the lenses, with regard to transmittance variation, at the mean wavelength of every spectral range i.e. $300 \mathrm{~nm}$ for UVB, $380 \mathrm{~nm}$ for UVA and $600 \mathrm{~nm}$ for visible range. As we can observe, although the differences between worn and unworn contact lenses are small, the transmittance, in general, decreased for worn contact lenses. The values changes from $1 \%$ to $10 \%$ in the UVB wavelengths, from $1 \%$ to $7 \%$ in the UVA wavelengths and from $2 \%$ to $6 \%$ in the visible light spectrum. In this table there is one value at which the transmittance increases slightly in the band UVB and two values in the band UVA but these differences are not statistically significant and may be due to fluctuations in the source emission.

It should be considered the possibility that the variety and amount of proteins adsorbed onto the contact lens could have been influenced by the lens material or by the lens care solution used. "In vitro" dehydration studies have revealed that conventional hydrogel contact lenses are more prone to dehydratation than silicone hydrogel contact lenses, so, regardless of their hydrophilicity, conventional hydrogel contact lenses adsorb more proteins than the other lenses, even if worn for a shorter period of time (15 days) ${ }^{15,16}$.

The interaction between a contact lens, the ocular surface, and the tear film is vital for successful contact lens wear. It has long been known that both organic and inorganic tear film and ocular surface-related components deposit on contact lenses. Over the years, significant emphasis has been placed on understanding the interaction between protein and lipid deposits on contact lenses. It has become well recognize that the polymer composition of the contact lens material has a significant impact on the trends associated with deposition patterns. Likewise, other factors such as lens care, compliance, and the environmental all may play a role in observed trends in deposition pattens ${ }^{17}$.

The ocular surface environment is very complex, and tear film composition varies greatly between individuals. It is known that silicone hydrogel lenses attract greater amounts of lipid compared to many polyHema-based lenses which is the opposite trend to that seen with protein accumulation. Increased lipid deposition may impact clarity of vision, wettability and comfort, but has not been associated directly with ocular surface inflammatory responses ${ }^{18}$. 
In conclusion, this work demonstrates that transmittance of contact lenses Biofinity (CooperVision) is modified after the lenses being worn and these differences are more noticeable in the UVB spectrum. Although this, the value of visual acuity did not decrease in any of the patients to compare the values between the first day of use of contact lenses and the last. A possible explanation for this is that the multipurpose disinfecting solution has also influenced on the results obtained and the vision is only degraded when a considerable deposition occurs or when the denaturation of the adsorbed proteins is significant ${ }^{10}$.

Table 2. Transmittance of the contact lenses evaluated for the mean wavelength of each spectral range.

\begin{tabular}{c|cc|cc|c|c|} 
& \multicolumn{7}{|c|}{ Transmittance \pm MAD) (\%) } \\
Power & \multicolumn{2}{|c|}{ UVB (300 nm) } & \multicolumn{2}{|c|}{ UVA (380 nm) } & \multicolumn{2}{|c|}{ Visible (600 nm) } \\
$\mathbf{( D )}$ & Unworm & Worn & Unworm & Worn & Unworm & Worn \\
$\mathbf{- 1 , 5 0}$ & $89 \pm 1$ & $\mathbf{8 8} \pm \mathbf{1}$ & $91 \pm 1$ & $\mathbf{9 3} \pm \mathbf{1}$ & $93 \pm 2$ & $\mathbf{9 3} \pm \mathbf{1}$ \\
$\mathbf{- 1 , 7 5}$ & $89 \pm 2$ & $\mathbf{8 6} \pm \mathbf{1}$ & $94 \pm 1$ & $\mathbf{9 3} \pm \mathbf{2}$ & $95 \pm 1$ & $\mathbf{9 3} \pm \mathbf{1}$ \\
$\mathbf{- 2 , 2 5}$ & $91 \pm 2$ & $\mathbf{8 7} \pm \mathbf{1}$ & $94 \pm 1$ & $\mathbf{9 3} \pm \mathbf{1}$ & $97 \pm 1$ & $\mathbf{9 4} \pm \mathbf{2}$ \\
$\mathbf{- 2 , 5 0}$ & $91 \pm 1$ & $\mathbf{8 8} \pm \mathbf{2}$ & $94 \pm 1$ & $\mathbf{9 0} \pm \mathbf{2}$ & $97 \pm 1$ & $\mathbf{9 3} \pm \mathbf{1}$ \\
$\mathbf{- 3 , 0 0}$ & $93 \pm 2$ & $\mathbf{8 7} \pm \mathbf{1}$ & $95 \pm 1$ & $\mathbf{9 1} \pm \mathbf{1}$ & $96 \pm 1$ & $\mathbf{9 4} \pm \mathbf{1}$ \\
$\mathbf{- 4 , 2 5}$ & $92 \pm 1$ & $\mathbf{8 2} \pm \mathbf{1}$ & $93 \pm 1$ & $\mathbf{8 9} \pm \mathbf{1}$ & $94 \pm 1$ & $\mathbf{9 2} \pm \mathbf{1}$ \\
$\mathbf{- 5 , 0 0}$ & $92 \pm 1$ & $\mathbf{8 8} \pm \mathbf{2}$ & $96 \pm 2$ & $\mathbf{8 9} \pm \mathbf{1}$ & $96 \pm 1$ & $\mathbf{9 0} \pm \mathbf{1}$ \\
$\mathbf{- 5 , 2 5}$ & $91 \pm 1$ & $\mathbf{8 3} \pm \mathbf{1}$ & $93 \pm 1$ & $\mathbf{9 0} \pm \mathbf{1}$ & $95 \pm 1$ & $\mathbf{9 2} \pm \mathbf{1}$ \\
$\mathbf{- 5 , 7 5}$ & $91 \pm 1$ & $\mathbf{8 5} \pm \mathbf{1}$ & $95 \pm 1$ & $\mathbf{9 0} \pm \mathbf{1}$ & $95 \pm 1$ & $\mathbf{9 1} \pm \mathbf{1}$ \\
$\mathbf{- 7 , 0 0}$ & $88 \pm 1$ & $\mathbf{8 8} \pm \mathbf{2}$ & $92 \pm 1$ & $\mathbf{9 1} \pm \mathbf{1}$ & $95 \pm 1$ & $\mathbf{9 1} \pm \mathbf{1}$ \\
$\mathbf{- 7 , 5 0}$ & $88 \pm 2$ & $\mathbf{8 9} \pm \mathbf{3}$ & $91 \pm 1$ & $\mathbf{9 2} \pm \mathbf{1}$ & $94 \pm 1$ & $\mathbf{9 2} \pm \mathbf{1}$
\end{tabular}

\section{CONCLUSIONS}

The silicone hydrogel contact lenses used in this study, Biofinity of CooperVision, and manufactured with Comfilcon-A present small transmittance differences between unworn and worn. In general, both in the ultraviolet range and in the visible, slight decrease in transmittance are observed for the worn contact lenses. The values changes from $1 \%$ to $10 \%$ in the UVB wavelengths, from $1 \%$ to $7 \%$ in the UVA wavelengths and from $2 \%$ to $6 \%$ in the visible light spectrum.

Transmittance is modified after contact lenses wear, probably due to the formation of bio films on the lens surface, but these changes observed in the UV-VIS spectrum seem not to have any implications in visual performance of these contact lenses demonstrating their transparency even after wear. The value of visual acuity did not decrease in any of the patients to compare the values between the first day of use of contact lenses and the last.

We have also found that lens power does not have a statistically effect on transmittance of this contact lenses. 


\section{ACKNOWLEDGMENT}

We thank CooperVision for supplying the contact lenses evaluated in this study.

This work was supported by the "Generalitat Valenciana" (Spain) under project PROMETEO/2011/021, ISIC/2012/013 and GV/2012/099 and by "Ministerio de Ciencia e Innovación” (Spain) under project FIS2011-29803-C02-02.

\section{REFERENCES}

[1] International Commission on Non-ionizing Radiation Protection (ICNIRP). Guidelines on limits of exposure to ultraviolet radiation of wavelengths between $180 \mathrm{~nm}$ to $400 \mathrm{~nm}$ (incoherent optical radiation). Health Phys 2004; 87:171-86. Available at: http://www.icnirp.de/documents/UV2004.pdf

[2] Harris M.G., Dang M., Garrod S., Wong W., "Ultraviolet transmittance of contact lenses," Optometry Vision Sci. 71(1), 1-5 (1994).

[3] Harris M.G., Haririfar M., Hirano K.Y., "Transmittance of tinted and UV-blocking disposable contact lenses," Optometry Vision Sci. 76(3), 177-80 (1999).

[4] Harris M.G., Chin R.S., Lee D.S., Tam M.H., Dobkins C.E., "Ultraviolet transmittance of the vistakon disposable contact lenses," Contact Lens and Anterior Eye 23(1), 10-5 (2000).

[5] Quesnel N.M., Simonet P., "Spectral transmittance of UV-absorbing soft and rigid gas permeable contact lenses," Optometry Vision Sci. 72(1), 2-10 (1995).

[6] Moore L., Ferreira J.T., "Ultraviolet (UV) transmittance characteristics of daily disposable and silicone hydrogel contact lenses," Contact Lens and Anterior Eye 29(3), 115-22 (2006).

[7] Merindano M.D., Canals M., Saona C., Potau J., Costa J., "Observation of deposits on disposable contact lenses by bio-, light- and scanning electron microscopy," Contact Lens and Anterior Eye 21(2), 55-9 (1998).

[8] Michaud L., Giasson C., "Comparing the extent of protein build-up on several disposable lenses by two spectrophotometric methods," Contact Lens and Anterior Eye 21(4), 104-8 (1998).

[9] Santos L., Rodrigues D., Lira M., Real Oliveira M.E.C.D., Oliveira R., Yebra-Pimentel Vilar E., Azeredo J., "The influence of surface treatment on hydrophobicity, protein adsorption and microbial colonisation of silicone hydrogel contact lenses," Contact Lens and Anterior Eye 30(3), 183-8 (2007).

[10]Lira M., dos Santos Castanheira E.M., Santos L., Azeredo J., Yebra-Pimentel E., Real Oliveira M.E.C.D., "Changes in UV-visible transmittance of silicone-hydrogel contact lenses induced by wear," Optometry Vision Sci. 86(4), 332-9 (2009).

[11] Walsh J.E., Koehler L.V., Fleming D.P., Bergmanson J.P.G., "Novel method for determining hydrogel and silicone hydrogel contact lens transmission curves and their spatially specific ultraviolet radiation protection factors," Eye and Contact Lens 33(2), 58-64 (2007).

[12] Holden B.A., Sankaridurg P., Jalbert I., "Silicone hydrogels-adverse events, the epithelium in extended wear and inflammation and infection in the closed eye," Contact Lens Monthly 219, 34-42 (2000).

[13] Tighe B., Brennan N., Coles C., "Silicone hydrogels-what are they and how should they be used in everyday practice?" Contact Lens Monthly 218, 31-5 (1999).

[14] StellarNet Fiber Optic Spectrometer Manual http://www.stellarnet-inc.com/

[15] Tranoudis I., Efron N., "Water properties of soft contact lens materials," Contact Lens Anterior Eye 27, 193208 (2004).

[16] Jones L., May C., Nazar L., Simpson T., "In vitro evaluation of the dehydratation characteristics of silicone hydrogel and conventional hydrogel contact lens materials," Contact Lens Anterior Eye 25, 147-56 (2002).

[17] Nichols J.J., "Deposition on silicone hydrogel lenses," Eye and Contact Lens 39, 20-23 (2013).

[18] Luennsmann D., Jones L. , "Protein deposition on contact lenses: The past, the present, and the future," Contact Lens Anterior Eye 35, 53-64 (2012). 